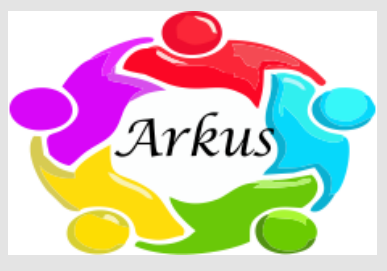

\title{
ARKUS
}

Journal Homepage:

https://hmpublisher.com/index.php/arkus

\section{Qur'an Recitation Therapy as Anxiety Treatment}

\section{Carla Raymondalexas Marchira ${ }^{1 *}$}

${ }^{1}$ Department of Psychiatry, Faculty of Medicine, Nursing and Public Health, Universitas Gadjah Mada, Yogyakarta, Indonesia

\author{
A R T I C L E I N F O \\ Keywords: \\ Anti-anxiety agents \\ Anxiety \\ Anxiety disorders \\ Emotions \\ Iatrogenic disease
}

\section{Corresponding author:}

Carla Raymondalexas Marchira

E-mail address:

\section{cmarchira@ugm.ac.id}

The author has reviewed and approved the final version of the manuscript.

https://doi.org/10.37275/arkus.v6i2.86

\section{A B S T R A C T}

Anxiety is an uncomfortable, pleasant, indistinct and pleasant feeling with autonomic symptoms. Many internal and external factors cause anxiety in a person. The human body tries to maintain homeostasis at all times. Anything that disrupts homeostasis is defined as a stressor by the body. To compensate for stressors and to restore homeostatic equilibrium, physiological adaptations occur in response to stress. This adaptation involves a hormonal cascade along the HPA axis, namely the release of corticotropin-releasing factor ( $\mathrm{CRF}$ ) which stimulates the release of adrenocorticotropic hormone (ACTH) and causes the release of stress hormones (glucocorticoids and epinephrine) from the adrenal cortex. Glucocorticoids will provide negative feedback (negative feedback) to the hypothalamus, thereby reducing the release of CRF. Non-pharmacological intervention anxiety that has lower side effects is safer and can be used for a more extended period compared to anti-anxiety drugs. The recitation of the Al-Qur'an generates alpha waves, which are waves that appear when someone is relaxing. Increased alpha waves can reduce symptoms of anxiety.

\section{Introduction}

The American Psychiatric Association (APA) defines anxiety as a psychological and physiological state characterized by cognitive, physiological and behavioural components that are described as anticipating future dangers and accompanied by symptoms of dysphoria. Meanwhile, the International Classification of Disease (ICD-10) defines anxiety as a feeling of worry, motor tension manifested by restlessness or muscle tension, and excessive autonomic activity such as headaches or sweating. 1

Health conditions are often associated with anxiety. Anxiety can be both a cause and a result of the disease. Patients with chronic diseases often experience anxiety disorders, and patients with anxiety disorders tend to be more susceptible to diseases such as infections, allergies and asthma. Gastrointestinal disorders and migraines are the most common diseases and are strongly associated with anxiety. 1

Anxiety is different from fear. Anxiety is a response to a threat to something unknown, comes from within the individual, is vague and conflictual. In contrast, fear is a response to a known threat, comes from outside the individual and does not cause conflict.

The non-pharmacological intervention of anxiety 
is believed to have lower side effects, is safer and can be used for a more extended time compared to antianxiety drugs. The recitation of Holy Qur'an generates alpha waves, which are waves that appear when a person relaxes. Increased alpha waves can reduce symptoms of anxiety.

\section{Discussion}

Anxiety is a normal and adaptive response that saves lives by warning individuals against something that threatens bodily damage, pain, helplessness, physical and social needs. The body signals against this threat by increasing sympathetic and parasympathetic nervous activity which increases somatic and autonomic activity. However, this response can be categorized as an anxiety disorder if the symptoms are more than usual. ${ }^{2}$

In the $5^{\text {th }}$ edition of the Diagnostic and Statistical Manual of Mental Disorders (DSM), diagnoses included in the category of anxiety disorders include separation anxiety disorder, selective mutism, specific phobia, social phobia, panic disorder, agoraphobia, generalized anxiety disorder, substance/medication-induced anxiety disorder, and anxiety disorder due to another medical condition. In the $3^{\text {rd }}$ edition of the Practical Guidelines for the Diagnosis of Mental Disorders (PPDGJ), anxiety is included in the F40-F48 block classification of neurotic disorders, somatoform disorders and stressrelated disorders.

The prevalence of anxiety in women is higher than that of men. ${ }^{2}$ Women experience anxiety earlier than men. A prospective study found a sharp increase in anxiety rates in five-year-old girls. However, no gender differences were found in the mean age at the onset or duration of anxiety disorder.

Life experiences play a role in anxiety disorders, especially phobias and panic disorders. Posttraumatic stress disorder originates from catastrophic events (catastrophic). A stressful life can trigger panic episodes and be a common cause of anxiety disorder. ${ }^{1}$ A negative assessment of stressful life events is also a risk factor for any anxiety disorder. 4

Low self-esteem is related to anxiety disorders, especially social anxiety disorders. A person with low self-esteem trusts the opinions of others more than he does, which makes it challenging to evaluate himself and creates anxiety. 5

Family history of suffering from psychological disorders is also a risk factor. In twins, if one of the identical twins has a psychiatric disorder, the risk of the other twin having the same condition is about $50 \%$. The role of the family in increasing the risk of anxiety is disharmony in the family. 5

The next factor that can trigger anxiety is work. The high psychological demands in the workplace in the form of low social support, emotional demands, role conflicts, ethical conflicts, tensions with the public, and job security increase the risk of overall anxiety disorders. ${ }^{4}$ Alcohol consumption and smoking can increase the risk of anxiety. Women who consume alcohol more than 14 times a week are at twice the risk of experiencing anxiety than women who drink little alcohol. However, this does not apply to men who have high drinking levels 21 times a week. Smoking one pack per day can increase the risk of anxiety disorder type generalized anxiety disorder, agoraphobia by six times and panic disorder by two times. 4

There is no definite aetiology to cause anxiety and disturbance. Anxiety is believed to emerge as a combination of biological, psychological and social factors. The symptoms of anxiety disorder are thought to be the result of a disruption in the balance of activity in the brain's emotional centre, namely the limbic system. Apart from the limbic system, the hypothalamic-pituitary-adrenal (HPA axis) axis also acts as a human physiological response to stress. The mediators that are considered to be intermediaries are norepinephrine, serotonin, dopamine, glutamate and GABA. ${ }^{6}$ 
The human body tries to maintain homeostasis at all times. Anything that disrupts homeostasis is defined as a stressor by the body. To compensate for stressors and to restore homeostatic equilibrium, physiological adaptations occur in response to stress. This adaptation involves a hormonal cascade along the HPA axis, namely the release of corticotropinreleasing factor $(\mathrm{CRF})$ which stimulates the release of adrenocorticotropic hormone (ACTH) and causes the release of stress hormones (glucocorticoids and epinephrine) from the adrenal cortex. Glucocorticoids will provide negative feedback (negative feedback) to the hypothalamus, thereby reducing the release of CRF.

One of the stressors is when an individual feels his survival is being threatened and perceives it as a challenge for homeostasis. Suppose this condition persists and the individual mistrusts the homeostatic challenges that occur. In that case, the hormonal cascade in the HPA axis will take place and enter the fields of neurosis, anxiety and paranoia.

The primary modulator of fear or anxiety is the amygdala. The role of the amygdala is to identify emotions from stressors and to create emotional memories. The amygdala receives input from neurons in the cortex. The amygdala also receives sensory input which bypasses the cortex and thus tends to be subconscious. This fact is one reason sexual harassment victims feel suddenly anxious when interacting with friendly people. It took them a moment to realize that these individual characteristics reminded them of the person who had abused her. 7

Patients with anxiety disorders show an increased amygdala response. A study using functional magnetic resonance imaging (fMRI) demonstrated hyperactivity of the amygdala during provocation of symptoms associated with fear. 8

Individuals with social anxiety disorder show increased amygdala responses to emotional and nonsocial stimuli compared to a healthy control group. Levels of social anxiety correlated positively with amygdala reactivity.

The structures of the amygdala and other limbic systems are connected to regions of the prefrontal cortex. The prefrontal-limbic activation disorder is reversible by psychological or pharmacological intervention. The amygdala can also activate the midbrain and brainstem, which causes autonomic hyperactivity and correlates with physical symptoms of anxiety. Thus, the hyperactive HPA axis is associated with the onset of depression and anxiety disorders. 9 The amygdala functions to increase HPA axis activity, while the hippocampus has a function to suppress the activity of the HPA axis.

$\mathrm{CRF}$ is a neurotransmitter that acts as a significant mediator of autonomic, behavioural, immune, and endocrine stress responses. CRF can be anxiogenic, depressogenic, proinflammatory and improve pain perception. GABA inhibits CRF release. Glucocorticoids activate the locus coeruleus which sends projections to the amygdala via norepinephrine. The amygdala stimulates the release of large amounts of CRF, resulting in glucocorticoid secretion. This repeated stimulation results in communication that strengthens the synapses with other brain regions. Continuous exposure to glucocorticoids can deplete norepinephrine levels in the locus coeruleus. Norepinephrine is an important neurotransmitter involved in attention, alertness, motivation, and activity so that if the norepinephrine levels are reduced, depression can occur.

Serotonin is also involved in the pathogenesis of anxiety disorders. The increased serotonin can stimulate the hippocampal receptors, thereby increasing neuroprotection and neurogenesis, which has an anxiolytic effect. GABA is a major inhibitory neurotransmitter in the central nervous system that is believed to be closely linked in the pathophysiology of anxiety disorders. Benzodiazepines facilitate the transmission of GABA neurons and can exert a calming effect. 
The clinical manifestation of anxiety disorder is a sympathetic nervous response that triggers changes in the three-body function systems: cognitive, physical and attitude. Generalized anxiety disorder is usually characterized by generalized, persistent and unrestricted concern over a specific environment that occurs in weeks to months. Cognitive changes include a feeling that something is wrong, feeling crazy about the worries are experiencing and anxiety in the future. Physical symptoms include muscle tension, restlessness, and sleep disturbances. Attitude changes include avoiding reading the news and interrupting daily activities due to worries that occur. 10

The manifestation of panic disorder is a condition where autonomic symptoms accompany severe anxiety attacks, recur, sudden onset and last for approximately one month. The peak of a panic attack can occur within a few seconds to 10 minutes. Apart from shortness of breath, individuals experience panic attacks also feels tremors, dizziness, feeling cold, depersonalization and derealization. Another characteristic of panic disorder is that the patient will tend to avoid situations or places where panic attacks have or have occurred, especially places of social activity. Besides, individuals with panic disorder tend to avoid long trips and strenuous activities such as sports.

In social phobia anxiety disorder, cognitive changes that occur in the form of feelings of being unreliable, strange and anxious. Symptoms that arise include sweating, dry mouth and a flushed face. Individuals with social phobia tend to stay away from meetings, parties, family gatherings and public speaking. Specific phobic disorders usually experience cognitive changes during certain situations that they fear such as meeting animals, being at high altitudes, and seeing blood. Symptoms that arise include sweating, muscle tension and dizziness. Individuals with specific phobias tend to avoid things they fear, such as avoiding flying aeroplanes and avoiding zoos.

The cognitive changes found in individuals with post-traumatic stress disorder are feelings that the individual has never survived and that those around them cannot be trusted completely. Physical symptoms include sleep disturbances, nightmares, angry outbursts to excessive awareness of danger. Individuals with post-traumatic stress disorder tend to show an attitude of avoiding thoughts, feelings, conversations, activities, places or people involved with the traumatic event. 10

Non-pharmacological management given to patients with anxiety disorders varies. There are many studies on non-pharmacological interventions that have been shown to improve symptoms of anxiety ${ }^{11}$. Non-pharmacological therapy is believed to have lower side effects, is safer and can be used for a more extended period compared to anti-anxiety drugs.

Holy Qur'an recitation deals with spirituality. Spirituality involves cognitive and emotional a person such as beliefs, motivation and feelings of being close to God. Mental and emotional states are divided into positive states (faithful or believing in God) and negative states (judgments that God is punishing, God is unjust and death anxiety). However, several studies have found that individuals with unfavourable circumstances tend to have experienced anxiety disorders before. Cognitive therapy that aims to increase inner spirituality (involves adherence to fundamental spiritual values) is believed to be a therapeutic consideration for the individual.

\section{Conclusion}

Holy Qur'an recitation has a relaxing effect which can reduce symptoms of anxiety.

\section{References}

1. Tsuang MT, Tohen M, Jones PB. Textbook of Psychiatric Epidemiology. John Wiley \& Sons; 
2011.

2. Sadock BJ, Sadock VA, Ruiz P. Synopsis of Psychiatry. eleventh.; 2007.

3. Maslim R. Diagnosis Gangguan Jiwa, Rujukan Ringkas PPDGJ-III Dan DSM-5. Jakarta: PT Nuh Jaya; 2013.

4. Zimmermann M, Chong AK. Modifiable Risk and Protective Factors for Anxiety Disorders Among Adults: A Systematic Review. Psychiatry Res. 2019:112705. doi:10.1016/j.psychres.2019.112705

5. Blanco C, Ph D, Rubio J, et al. Risk Factors for Anxiety Disorders: Common and Specific Effects in a National Sample. Depress Anxiety. 2014;31(9):756-764. doi:10.1002/da.22247.RISK

6. Martin E, Ressler K, Binder E, Nemeroff C. The Neurobiology of Anxiety Disorders: Brain Imaging, GenMartin, E., Ressler, K., Binder, E., \& Nemeroff, C. (2013). The Neurobiology of Anxiety Disorders: Brain Imaging, Genetics, and Psychoneuroendocrinology. Psychiatr Clin North Am., 32(3), 549-575. ht. Psychiatr Clin North Am. 2009;32(3):549-575. doi:10.1016/j.psc.2009.05.004.The
7. Shelton CI. Diagnosis and Management of Anxiety Disorders. J Am Osteopath Assoc. 2004;104(3):2-5.

8. Fredrikson M, Faria V. Neuroimaging in anxiety disorders. Mod Trends Pharmacopsychiatry. 2013;29:47-66. doi:10.1159/000351938

9. Suma CP, Raman M. Anxiety. StatPearls.

10. Rector NA, Bourdeau D, Kitchen K, Massiah LJ. Anxiety Disorders an Information Guide.; 2011.

11. Saeed SA, Cunningham K, Bloch RM. Depression and Anxiety Disorders: Benefits of Exercise, Yoga, and Meditation. Am Fam Physician. 2019.

12. Chandler SK, Robins JL, Kinser PA. Nonpharmacologic interventions for the selfmanagement of anxiety in Parkinson's disease: A comprehensive review. Behav Neurol. 2019;2019. doi:10.1155/2019/8459579

13. Rosmarin DH, Leidl B. Spirituality, Religion, and Anxiety Disorders. INC; 2020. doi:10.1016/b978-0-12-816766-3.00003-3 